\title{
Breus' mole/chorangiosis/chorangioma of the placenta: a dilemma with a rare fetal outcome report
}

\author{
Nitika Sobti ${ }^{1 *}$, Ankita Chandna ${ }^{2}$, Bhawna Narula ${ }^{3}$
}

\begin{abstract}
${ }^{1}$ Department of Obstetrics and Gynecology, Max Health Care, Gurugram, Haryana, India
${ }^{2}$ Department of Obstetrics and Gynecology, Max Super Specialty Hospital, Shalimar Bagh, New Delhi, India

${ }^{3}$ Department of Pathology, Max Super Specialty Hospital, Shalimar Bagh, New Delhi, India
\end{abstract}

Received: 21 October 2019

Revised: 23 November 2019

Accepted: 03 December 2019

\author{
*Correspondence: \\ Dr. Nitika Sobti, \\ E-mail: drnitikasobti@gmail.com
}

Copyright: (C) the author(s), publisher and licensee Medip Academy. This is an open-access article distributed under the terms of the Creative Commons Attribution Non-Commercial License, which permits unrestricted non-commercial use, distribution, and reproduction in any medium, provided the original work is properly cited.

\begin{abstract}
Massive Subchorionic Thrombohematoma (MST) is a rare condition in which there is a massive collection of blood between the placental membranes and uterine wall separating the villous chorionic plate from villous chorion. It is relatively rare and is poorly understood. Many theories have been proposed to explain the etiology of Breus mole; some suggest it is a fetal haemorrhage, while others claim it has a maternal-origin thrombosis of placental vessels. A 30-year-old healthy Indian pregnant woman was presented at Max Hospital, Shalimar Bagh Delhi, India, during her second pregnancy with a complaint of fever. On routine level-2 ultrasonography (USG) done at 18.6 weeks of gestation showed thick placenta. No fetal tumours or any other anomalies were noted on that scan which was followed by a detailed scan which confirmed a solitary mass arising from fetal side $103 \times 64 \times 82 \mathrm{~mm} \mathrm{~S} / \mathrm{O}$ chorioangioma. Serial growth and doppler USG were conducted to monitor placental function, tumor characteristics and future anatomy. The subject received steroids to enhance fetal lungs maturation at Week 30, iron/calcium supplements, Ecosprin tablets, and progesterone support. At 32.5 weeks, the subject developed deranged sugars followed by gestational hypertension at 34.1 weeks. Ultrasonography also showed fetal growth restriction with large chorioangioma. The subject underwent a successful elective caesarean section at 34.4 weeks. On placental examination, $10 \mathrm{~cm}$ large mass encasing $3 / 4$ of the placenta was identified as a large subchorionic hematoma/chorioangioma $(800 \mathrm{~g})$. This study concludes that early identification of a large chorangioma aids in consequent fetal surveillance, management of maternal symptoms, and delivery planning discussions even if the pathological diagnosis turns out to be Breus' mole with underlying chorangiosis postnatally.
\end{abstract}

Keywords: Breus' mole, Chorioangioma, Chorangiosis, Perinatal outcomes, Placenta

\section{INTRODUCTION}

The placenta is the largest organ which is the mirror of maternal and foetal status. It is important for appropriate maternal and fetal development and functions. Therefore, histological examination of the placenta provides sensitive, specific, and scientific information for evaluating the nature and extent of the placental injury.
Massive subchorionic thrombohematoma (MST) is a rare condition in which there is a massive collection of blood between the placental membranes and uterine wall separating the villous chorionic plate from villous chorion. ${ }^{1,2}$ It was first termed Breus' mole and described in 1892. The frequency of MST is reported between $0.03 \%-0.08 \%$; its etiology and pathogenesis are yet unknown. The outcomes of MST are dependent on its site 
and size. If MST is near the cord insertion, it can lead to umbilical venous obstruction, cord compression, and decreased fetal perfusion. Therefore, MST is related to complications including fetal growth restriction (FGR) and intrauterine fetal death resulted due to placental insufficiency. 3,4

In some cases, there is a placental change that results in excessive formation of blood vessels within the terminal chorionic villi called chorangiosis. ${ }^{1,2}$ Presence of chorangiosis is associated with a high mortality rate $(39 \%)$ and congenital malformations $(42 \%) .{ }^{5}$ Differential diagnosis of chorangioma includes chorangiosis and chorangiomatosis, though in our case, it was diagnosed as Breus' mole with underlying chorangiosis. Therefore, it needs very close follow-up and management during the antenatal period for a successful fetal outcome. ${ }^{6}$

A case of an Indian female is reported with large placental chorioangioma on antenatal diagnosis, the antenatal management, and maternal and fetal outcome and the postnatal diagnosis.

\section{CASE REPORT}

A 30-year old healthy Indian pregnant woman with fever was presented at Max hospital, Gurugram, India, during her second pregnancy. She was evaluated by a level 2 ultrasonography (USG) at 18.6 weeks of gestation that showed placentomegaly. There were no fetal tumours or any other anomalies noted on that scan followed by a USG for monitoring growth which showed bulky placenta with a discrete hypoechoic mass S/O large chorioangioma. The ultrasound done at 31.4 weeks confirmed a solitary mass arising from fetal side $103 \times 64$ $\times 82 \mathrm{~mm} \mathrm{~S} / \mathrm{O}$ chorioangioma (Figure 1).

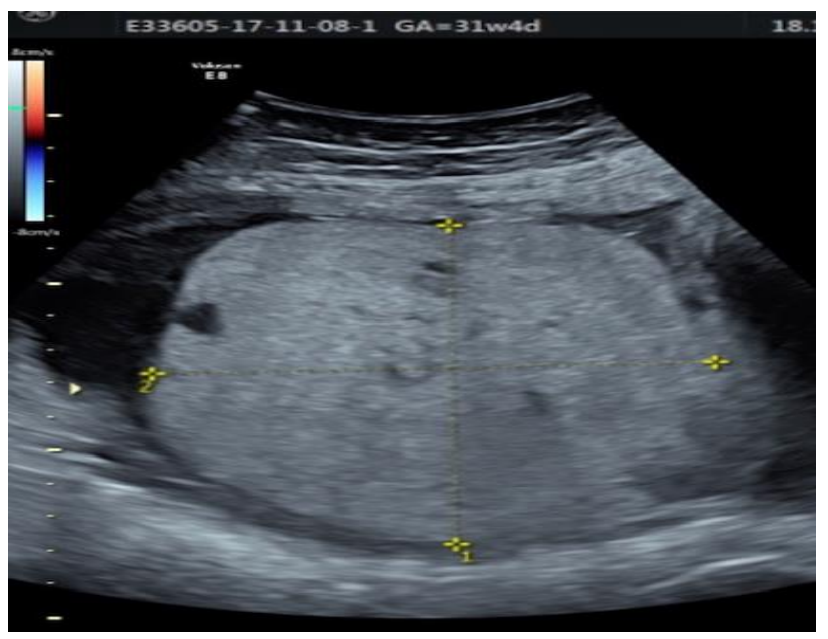

Figure 1: Ultrasound examination of the uterus at 31.4 weeks of gestation.

Serial growth and doppler USG were conducted to monitor placental function, tumor characteristics and future anatomy. In past, the subject had regular menstrual cycles, and the earlier antenatal period was normal. Two years back, the subject had uneventful caesarean delivery for breech and had a healthy female child.

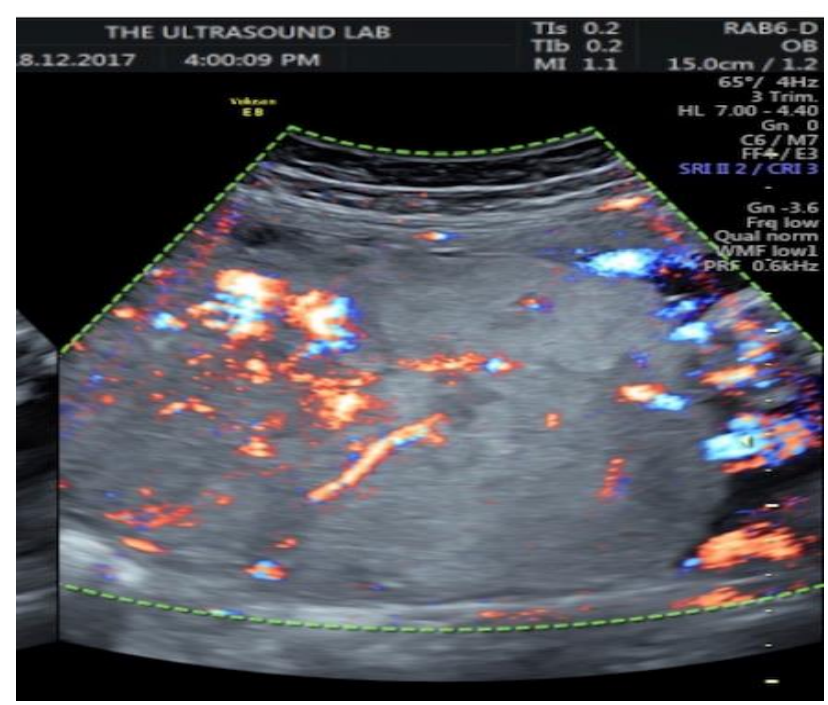

Figure 2: Ultrasound examination of the uterus after week 34 of gestational age.

The subject was counselled and kept under strict fetalmaternal surveillance. Fetal medicine and genetic consultation were also sought. Weekly USGs were done to see for any increase in the size of the placental tumor and to monitor fetal growth. The subject received steroids to enhance fetal lungs maturation at week 30 . She was advised to take iron/calcium supplements, ecosprin tablets, and progesterone support. At week 32.5, the subject developed deranged sugars, and at week 34.1, she developed gestational hypertension which was managed conservatively. Serial USG showed FGR with large chorioangioma (Figure 2). In view of high BP readings with deranged sugars and FGR, the decision to deliver the baby by caesarean section was taken in consultation with neonatologist. The subject underwent a successful elective caesarean section at week 34.4 and delivered a healthy female child of $1.89 \mathrm{~kg}$ with an Apgar score of 8 , 9,8 . She was shifted to neonatal intensive care unit for observation. After the operation, was BP and blood sugar of the subject were within the normal ranges. Both mother and baby were discharged on day 5 .

Gross examination revealed a large nodular cystic lesion (blood clot) measuring $10 \times 5 \mathrm{~cm}$ covering a large portion of the placental disc on the fetal surface (Figure 3 and Figure 4). Microscopic examination revealed an intervillous hematoma and fibrinous deposits directly beneath the chorionic plate with chorangiosis (increased vessels in terminal villi; (Figure 5). A large amount of blood was stored in the subchorionic space. No hematoma or infarction was observed on the maternal surface of the placenta. Based on these findings, MST with chorangiosis was diagnosed. It was recommended chromosomal analysis of the baby as well which the 
subject was not willing to do. The subject had an uneventful postoperative course.

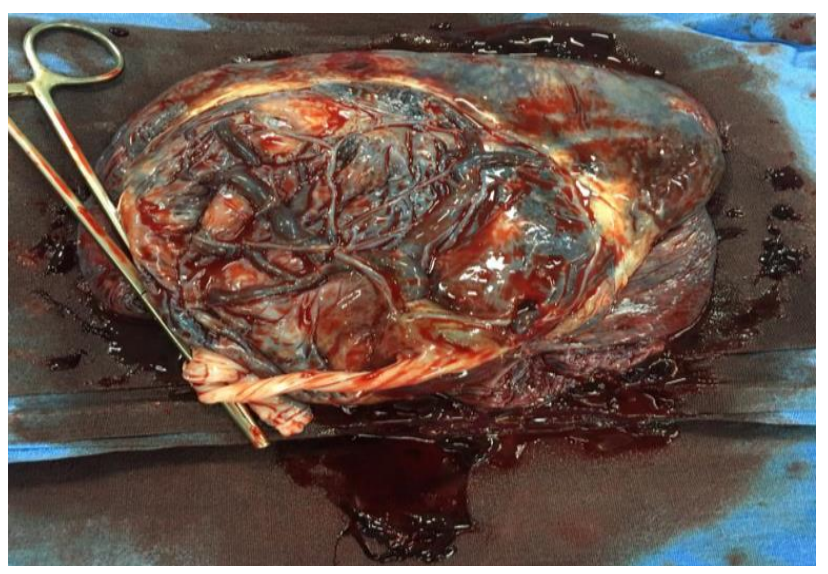

Figure 3: Large nodular cystic lesion.

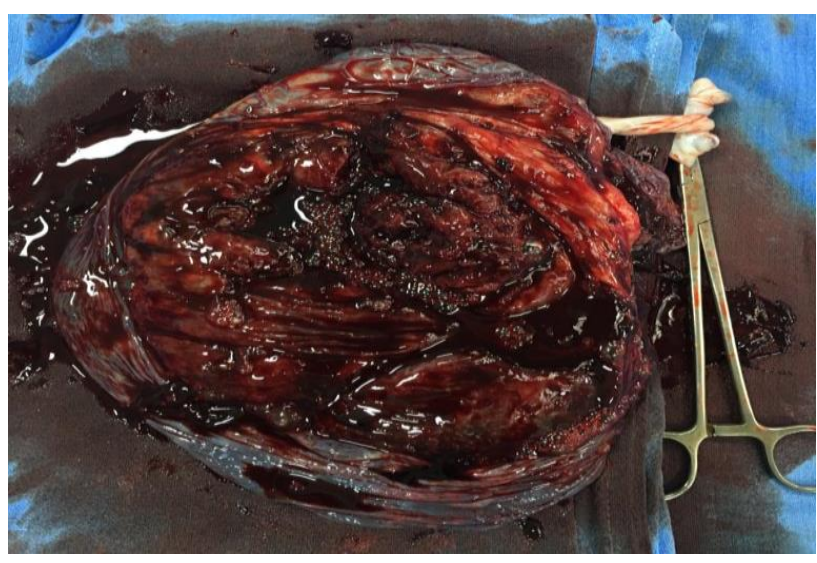

Figure 4: Cut section of large nodular cystic lesion.

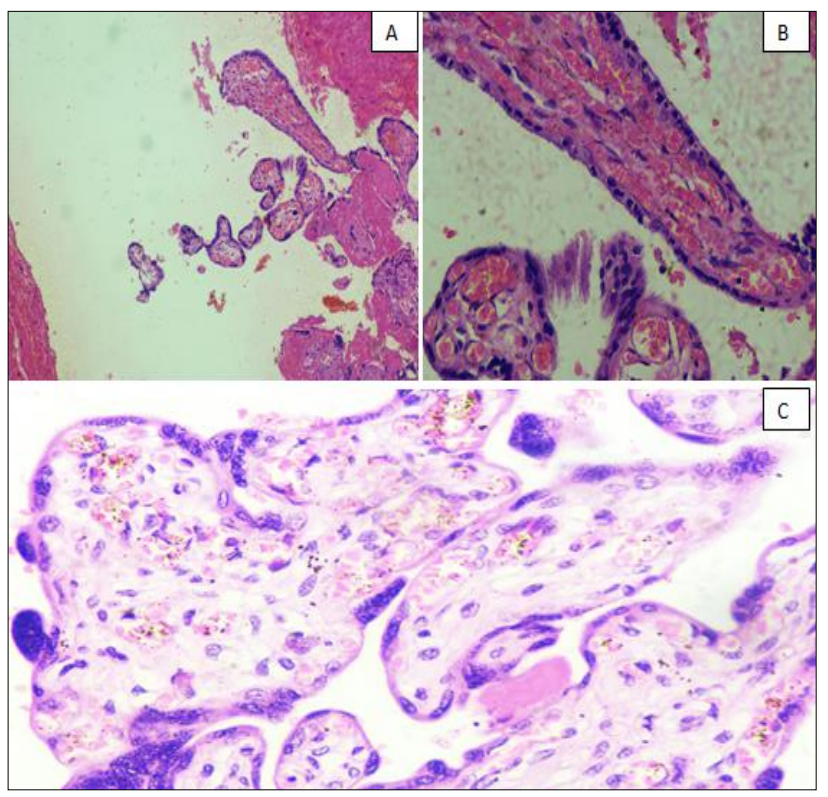

Figure 5: Microscopic examination of umbilical cord A) at low magnification B) at medium magnification C) at high magnification.

\section{DISCUSSION}

This was a case report study at Max Hospital, Gurugram, India, on a 30-year-old female subject who reported an MST, a rare condition with underlying chorangiosis. Chorangiosis is a rare placental vascular anomaly which is hypoxia-related angiogenesis. Both conditions can be associated with increased FGR and intrauterine fetal death. $^{7}$ The diagnosis is often made with histopathological examination of placenta after delivery.

Massive subchorionic thrombohematoma may be reported as homogenous, heterogeneous, or hypoechogenic mass in the chorion. Its appearance is different from the ultrasonic appearance of normal placental tissue, and can occur as placentomegaly. ${ }^{3}$ In a study, elastography showed clearly the differentiation of a hematoma from the placenta in relation to a case of possible placenta previa. ${ }^{8}$ However, with USG prenatal, diagnosis is generally challenging. In our case, the diagnosis of MST with USG was not possible because of severe oligohydramnios. Though, magnetic resonance imaging (MRI) results of MST vary, MRI is reported useful in differentiating MST from rest of the placental diseases, such as chronic abruption oligohydramnios sequence, placental abruption, and placental mesenchymal dysplasia. A high signal at the rim on T1weighted images represented thrombohematoma while a low signal at the marginal zone on the T1- and T2weighted images represented it. In addition, steady-state free precession MRI is used to show the arrangement of the placenta, umbilical cord, hematoma, and foetus clearly. In a previous study, of 14 subchorionic hematomas, 9 were confirmed by MRI.9,10 Overall, these results suggest that MRI is optimal for the diagnosis of MST when USG diagnosis is difficult. The MST etiology remains obscure. In MST, a large blood amount, especially of maternal origin, collects and separates the chorionic plate from the villous chorion. DNA analysis revealed that a large amount of the blood $(85 \%)$ in the thrombus is of maternal origin. ${ }^{11}$ Fetal villous haemorrhage or obstruction with further accumulation of blood and separation of the chorionic plate massively is proposed to cause MST. ${ }^{12}$ Further, maternal blood stasis in the subchorionic space causes thrombosis. ${ }^{3,4}$ Recently MST is reported following thrombolytic therapy and in patients with thrombophilic conditions. ${ }^{13}$ Chorangiosis is a rare condition and found in up to $5 \%$ of pregnancies. A large study reported chorangiosis with the incidence of 3\% among 1614 deliveries which was associated with placental lesions including foetal artery thrombosis, infarcts, and chronic villitis. ${ }^{14,15}$ Villous hypervascularity in which individual terminal villi comprises vessels in excessive numbers has been classified as chorangiosis. There should be $>10$ terminal villi containing $>10$ capillaries per villous in 10 medium power fields occurring in multiple areas (minimum 3 ) of the placenta. ${ }^{5}$

Uteroplacental insufficiency is the basic mechanism of FGR. In severe FGR cases, at least half of the surface of 
the placenta could be covered with haemorrhage under the chorionic plate. In such instances, the finding of normal uterine artery doppler waveforms at presentation is a favorable sign (prognostic) of perinatal survival. In addition, in MST, the site of severe haemorrhage is important whether entirely resides within the membranes and faraway from the placental disc or detach implantation of placenta. ${ }^{4,16}$ In our case, the subject had FGR with gestational diabetes, gestational hypertension and anaemia. Grossly there was placentomegaly and large nodular cystic lesion ( blood clot) measuring $10 \times 5 \mathrm{~cm}$ covering a large portion of the placental disc on the fetal surface with microscopic features of chronic villitis. Massive subchorionic thrombohematoma is related to poor pregnancy outcomes. Fung et al, reported that only six of ten pregnancies with MST resulted in a live birth, only 2 of which reached full term. Another study compared seven survivors and seven non-survivors with MST and concluded that MST can be diagnosed in the second trimester by USG examination of the placenta. ${ }^{4}$ In this case, the subject was diagnosed as having a large chorangioma about $10 \mathrm{~cm}$ encasing $3 / 4$ of the placenta with $1 \mathrm{~cm}$ in diameter. Though, on histological examination diagnosis of Breus' mole with underlying chorangiosis was made. The fetal outcome was normal, and the infant had weight $1.89 \mathrm{~kg}$ at birth. The infant was preterm with a gestational age of 34 weeks at birth. The management of gestational hypertension and FGR and preterm delivery was done for the successful fetal outcome. As shown in our case, Breus' mole placental chorioangiomas/chorangiosis are not always associated with bad outcomes. Study also favours that early diagnosis results in improved outcomes with close follow-up of affected pregnancies and use of therapeutic intervention as indicated in case complications arise. Although the incidence is low nearly 1 in 1200 placentae, detailed screening of placenta by USG preferably incorporating modalities such as colour flow imaging and MRI might allow better perinatal outcomes. ${ }^{17}$ Thus, we suggest that Breus' mole with chorangiosis, a marker of chronic hypoxia is associated with fetal and maternal complications like gestational diabetes, hypertension, severe anaemia, FGR, intrauterine death and congenital anomalies. Diagnosed it due to antenatal suspicion of a large chorioangioma and followed the subject with a successful subject outcome. This case study emphasizes the importance of comprehensive sonographic evaluation in pregnancy, especially in the presence of a suspected placental lesion.

Henceforth, obstetricians and pathologists should be aware of this entity and should send the placenta for pathological testing in suspected cases and should report this in the pathology report of each patient.

\section{CONCLUSION}

In general, Breus' mole occurs randomly without warning. It is important to share the diagnostic criteria of placental pathology, and radiological (antenatal) and histopathological (after delivery) along with close antenatal management for the successful fetal-maternal outcome. Great care should be taken in evaluating the entirety of the placenta. However, there is a potential for significant progression in size, vascularity and form which can result in late-onset fetal and maternal complications. Early identification of a placental (chorioangioma) aids in consequent fetal surveillance, management of maternal symptoms, and delivery planning discussions even if the pathological diagnosis turns out to be Breus' mole with chorangiosis postnatal. Till date to the best of our knowledge, the exact incidence of Breus' mole and chorangiosis has not been documented, and this uncommon and underreported entity has diagnostic implications. Its etiopathogenesis has remained a dilemma in the $18^{\text {th }}$ and $19^{\text {th }}$ century. The significance of Breus' mole does not depend upon the size but on their site of appearance. Therefore, early recognition of placental pathology and timely intervention is of utmost importance for a successful fetal outcome.

\section{Funding: No funding sources \\ Conflict of interest: None declared \\ Ethical approval: Not required}

\section{REFERENCES}

1. Gupta R, Nigam S, Arora P, Khurana N, Batra S, Mandal AK. Clinico-pathological profile of 12 cases of chorangiosis. Arch Gynecol Obstet. 2006;274(1):50-3.

2. Srinivasan AP, Omprakash BO, Lavanya K, Subbulakshmi Murugesan P, Kandaswamy S. A prospective study of villous capillary lesions in complicated pregnancies. J Pregnancy. 2014;2014:193925.

3. Fung TY, To KF, Sahota DS, Chan LW, Leung TY, Lau TK. Massive subchorionic thrombohematoma: a series of 10 cases. Acta Obstet Gynecol Scand. 2010;89(10):1357-61.

4. Alanjari A, Wright E, Keating S, Ryan G, Kingdom J. Prenatal diagnosis, clinical outcomes, and associated pathology in pregnancies complicated by massive subchorionic thrombohematoma (Breus' mole). Prenat Diagn. 2013;33(10):973-8.

5. Altshuler G. Chorangiosis. An important placental sign of neonatal morbidity and mortality. Arch Pathol Lab Med. 1984;108(1):71-4.

6. Agale SV, Dongaonkar DD. Chorangiosis of placenta. Bombay Hosp J. 2009;51(2):251-2.

7. Ingale YP, Buch AC, Ulhas PN, Kumar H. Incidental detection of chorangioma with chorangiosis of placenta: A rare case report. Med J DY Patil Vidyapeeth. 2019;12(2):174-176.

8. Ogawa M, Nagao D, Mori K, Sato M, Sato A, Shimizu D. Elastography for differentiation of subchorionic hematoma and placenta previa. Ultrasound Obstet Gynecol. 2012;39(1):112-4. 
9. Kojima K, Suzuki Y, Makino A, Murakami I, Suzumori K. A case of massive subchorionic thrombohematoma diagnosed by ultrasonography and magnetic resonance imaging. Fetal Diagn Ther. 2001;16(1):57-60.

10. Linduska N, Dekan S, Messerschmidt A, Kasprian G, Brugger PC, Chalubinski K. Placental pathologies in fetal MRI with pathohistological correlation. Placenta. 2009;30(6):555-9.

11. Kim DT, Riddell DC, Welch JP, Scott H, Fraser RB, Wright JR Jr. Association between Breus' mole and partial hydatidiform mole: chance or can hydropic villi precipitate placental massive subchorionic thrombosis? Pediatr Pathol Mol Med. 2002;21(5):451-9.

12. Fisteag-Kiprono L, Foster K, McKenna D, Baptista M. Antenatal sonographic diagnosis of massive subchorionic hematoma: a case report. J Reprod Med. 2005;50(3):219-21.

13. Heller DS, Rush D, Baergen RN. Subchorionic hematoma associated with thrombophilia: report of three cases. Pediatr Dev Pathol. 2003;6(3):261-4.
14. Aparna M, Archana J, Vidula G. Chorangiosis of placenta. Report of two cases. Panacea Journal of Medical Sciences. 2014;4(2):50-1.

15. FoxHarold, Sebire NJ. Abnormalities of villous vessels in pathology of placenta edited by Heather McCormick, Third edition. 2007;165-166.

16. Kingdom JC, Audette MC, Hobson SR, Windrim $\mathrm{RC}$, Morgen E. A placenta clinic approach to the diagnosis and management of fetal growth restriction. Am J Obstet Gynecol. 2018;218(2S):S803-S817.

17. Benirschke K. Abortions and moles. Monogr Pathol. 1981:23-48.

Cite this article as: Sobti N, Chandna A, Narula B. Breus' mole/chorangiosis/chorangioma of the placenta: a dilemma with a rare fetal outcome report. Int J Reprod Contracept Obstet Gynecol 2020;9:42731. 